\title{
Editorial
}

\section{Mathematical Modeling, Analysis, and Advanced Control of Complex Dynamical Systems}

\author{
Peng Shi, ${ }^{1}$ Hamid Reza Karimi, ${ }^{2}$ Xiaojie Su, ${ }^{3}$ Rongni Yang, ${ }^{4}$ and Yuxin Zhao ${ }^{5}$ \\ ${ }^{1}$ School of Electrical and Electronic Engineering, The University of Adelaide, Adelaide, SA 5005, Australia \\ ${ }^{2}$ Department of Engineering, Faculty of Technology and Science, University of Agder, 4898 Grimstad, Norway \\ ${ }^{3}$ College of Automation, Chongqing University, Chongqing 400044, China \\ ${ }^{4}$ School of Control Science and Engineering, Shandong University, Jinan 250061, China \\ ${ }^{5}$ College of Automation, Harbin Engineering University, Harbin 150001, China
}

Correspondence should be addressed to Peng Shi; peng.shi@adelaide.edu.au

Received 4 June 2014; Accepted 4 June 2014; Published 16 June 2014

Copyright (C) 2014 Peng Shi et al. This is an open access article distributed under the Creative Commons Attribution License, which permits unrestricted use, distribution, and reproduction in any medium, provided the original work is properly cited.

As most of technological environments or practical systems have a high complexity, complex dynamical systems have become the subject of intensive research in systems and control theory. The complexity of the system leads to severe difficulties that are encountered in the tasks of analyzing the system and designing and implementing control strategies algorithms. Here, the mathematical modeling and advanced control will provide a basis for the design and operation of complex dynamical systems, and these advanced techniques would result in potential and sustainable benefits.

This special issue contains four parts, that is, modeling and model approximation, stability analysis and robust control, filtering and state estimation, and engineering applications of complex dynamical systems. The contents of these parts are summarized as follows.

(1) Modeling and Model Approximation. "Frequency weighted model order reduction technique and error bounds for discrete time systems" by M. Imran et al. proposes a new frequency weighted technique for balanced model reduction of discrete time systems. The proposed technique guarantees stable reduced order models even for the case when two sided weightings are presented. "Modeling and optimal control of a class of warfare hybrid dynamic systems based on Lanchester $(n, 1)$ attrition model" by X. Chen and A. Zhang establishes a class of warfare hybrid dynamic systems by Lanchester equation in a $(n, 1)$ battle. This model can be characterized by the interaction of continuous-time models (governed by Lanchester equation) and discrete event systems (described by variable tactics).

(2) Stability Analysis and Robust Control. "Stability and $l_{1}$ gain control of positive switched systems with time-varying delays via delta operator approach" by $\mathrm{H}$. R. Karimi et al. investigates the problems of stability and $l_{1}$-gain controller design for positive switched systems with time-varying delays via delta operator approach. The purpose is to design a switching signal and a state feedback controller such that the resulting closed-loop system is exponentially stable with $l_{1}$-gain performance. "Localized and energy-efficient topology control in wireless sensor networks using fuzzy-logic control approaches" by J.-F. Martinez et al. aims at improving the network connectivity and fault-tolerant capability in response to node failures, while taking into account the fact that the control approach has to be localized and energy-efficient. Two fuzzy controllers are proposed in this paper: one is learning-based fuzzy-logic topology control, and the other one is rules-based fuzzy-logic topology control.

(3) Filtering and State Estimation. "Disturbance attraction domain estimation for saturated Markov jump systems with truncated Gaussian process" by K. L. Teo et al. studies the disturbance attraction domain estimation of saturated Markov jump systems with truncated Gaussian process. 
The problem of the optimal disturbance attraction domain is solved through searching for the most appropriate auxiliary parameters in the defined domain. "Recursive estimation for dynamical systems with different delay rates sensor network and autocorrelated process noises" by J. Feng solves the recursive estimation problem for a class of uncertain dynamical systems with different delay rates sensor network and autocorrelated process noises. The desired recursive robust estimators including recursive robust filter, predictor, and smoother are proposed by using the orthogonal projection theorem and an innovation analysis approach.

(4) Engineering Applications. "Command filtered adaptive fuzzy neural network backstepping control for marine power system" by X. Zhang and L. Mu designs a novel commandfiltered adaptive fuzzy neural network backstepping control method to retrain chaotic oscillation of marine power system. The main result, command-filtered adaptive fuzzy neural network backstepping control law, is presented for marine power system, and the Lyapunov stability theory is applied to prove that the system can remain closed-loop and asymptotically stable with this proposed controller. "Robust parametric control of spacecraft rendezvous" by $\mathrm{D}$. Gu and Y. Liu proposes a method to design the robust parametric control for autonomous rendezvous of spacecrafts. A novel concise control law for spacecraft rendezvous is given based on eigenstructure assignment and model reference theory. "Attitude analysis and robust adaptive backstepping sliding mode control of spacecrafts orbiting irregular asteroids" by C. Liang and Y. Li investigates attitude stability analysis and robust control algorithms for spacecrafts orbiting irregular asteroids with model uncertainties and external disturbances. "Rotor-flying manipulator: modeling, analysis, and control" by B. Yang et al. conducts the modeling, analysis, and control of the combined system, called rotor-flying multijoint manipulator (RF-MJM). The detailed dynamics model is constructed, and the full-state feedback linear quadratic regulator control problem is solved through obtaining linearized model near steady state.

Of course, the selected issues and papers are not a comprehensive representation of the area of this special issue. Nonetheless, they represent the rich and many-faceted knowledge that we have the pleasure of sharing with the readers.

\section{Acknowledgments}

We would like to express our appreciation to all the authors for their contributions. We also thank all the reviewers for their time and help in assessing all the submissions.

$$
\begin{array}{r}
\text { Peng Shi } \\
\text { Hamid Reza Karimi } \\
\text { Xiaojie Su } \\
\text { Rongni Yang } \\
\text { Yuxin Zhao }
\end{array}
$$




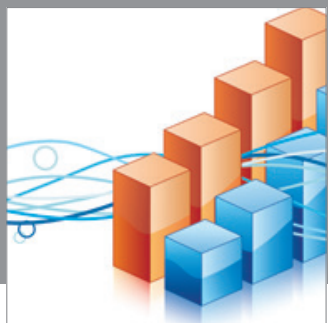

Advances in

Operations Research

mansans

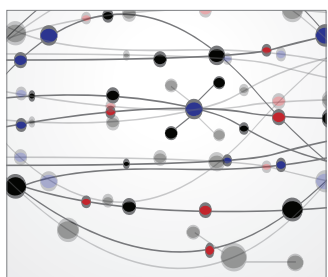

The Scientific World Journal
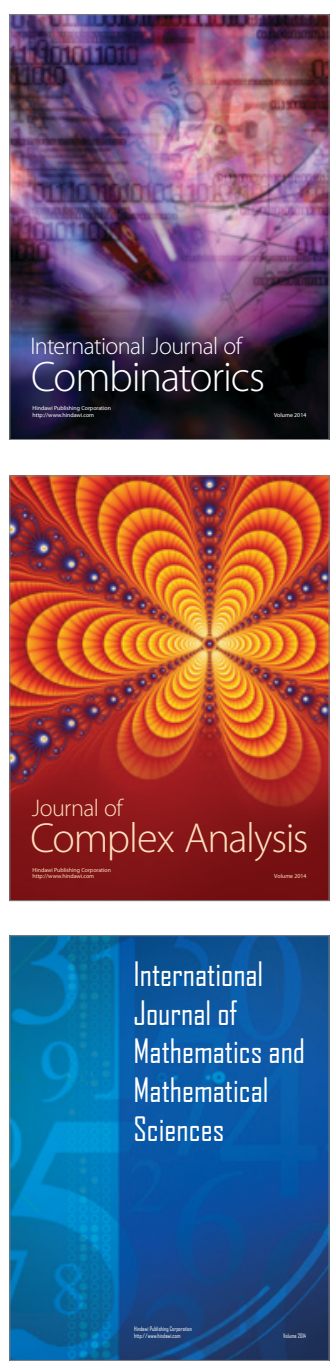
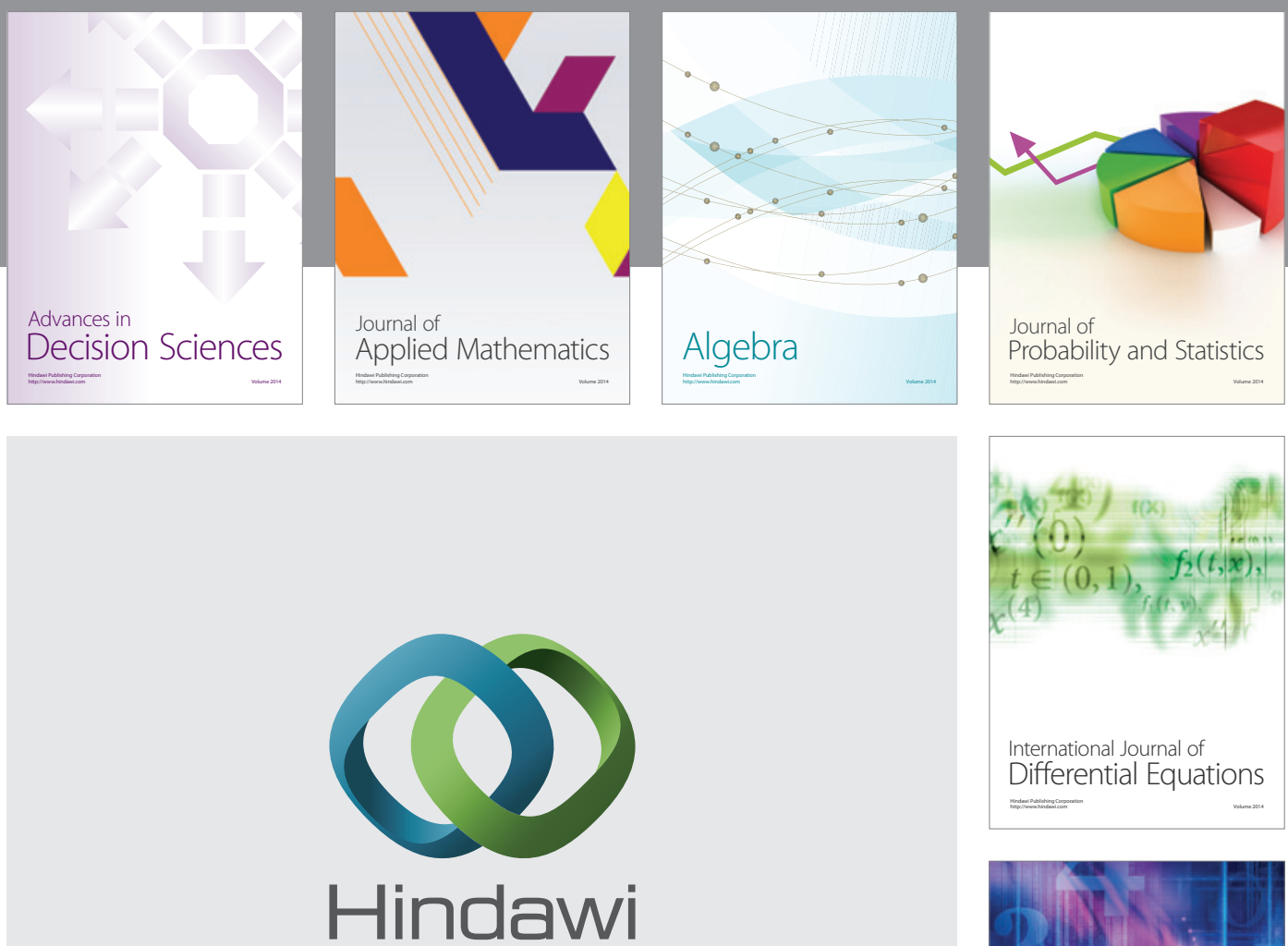

Submit your manuscripts at http://www.hindawi.com
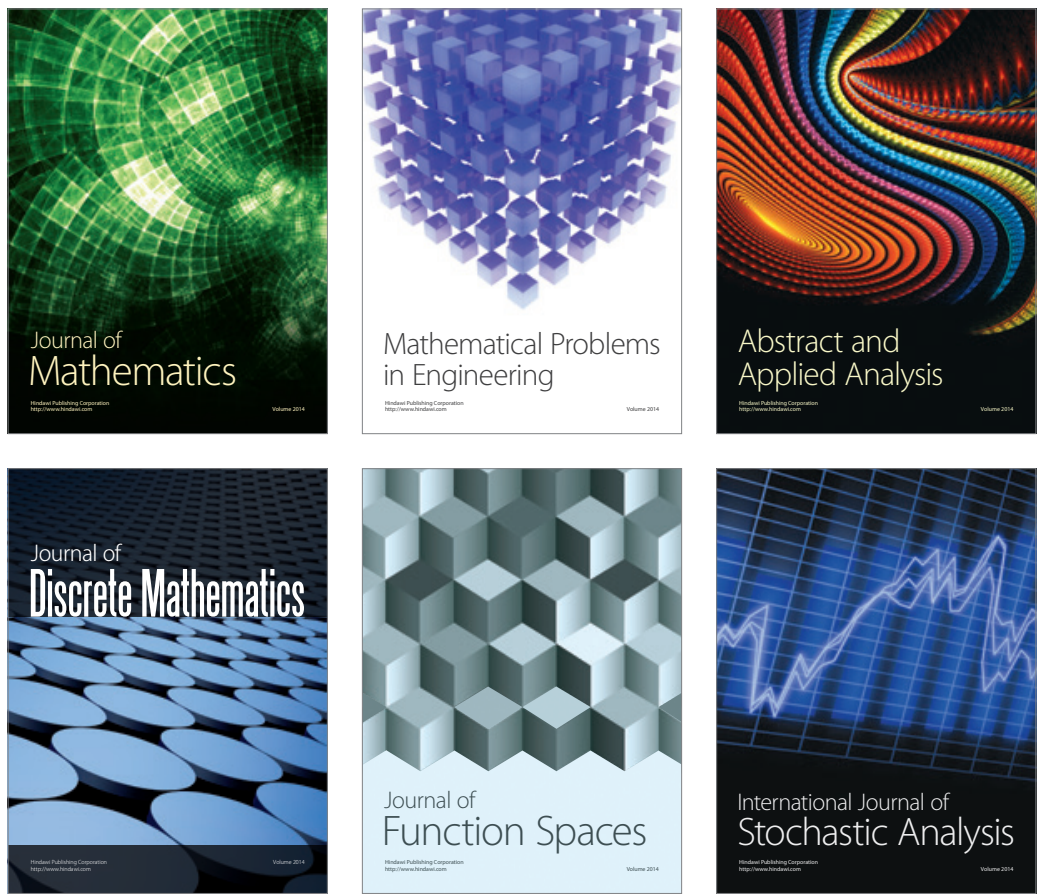

Journal of

Function Spaces

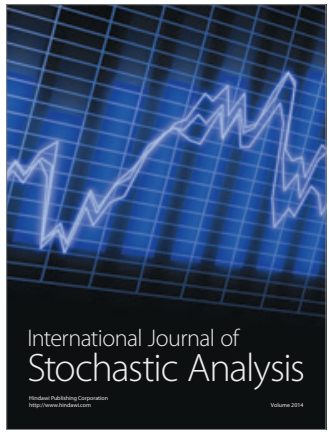

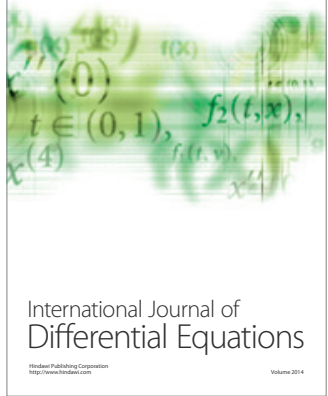
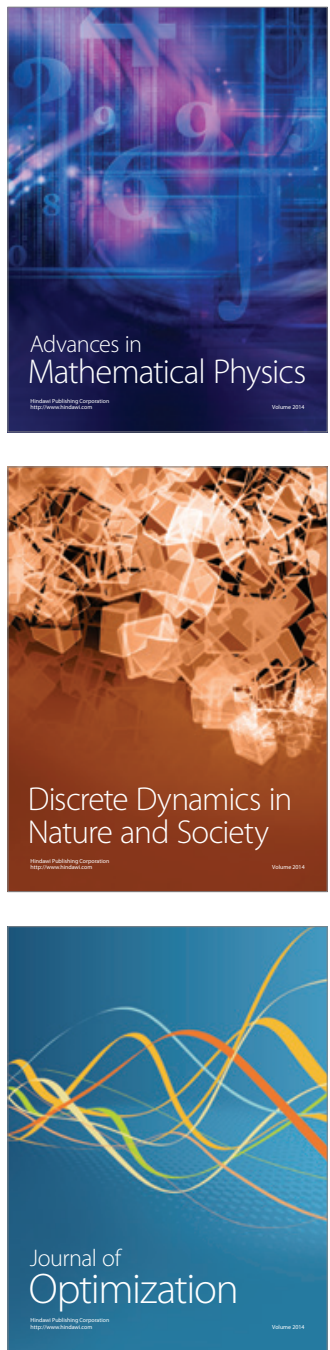